\title{
Importance of the subscapularis muscle after total shoulder arthroplasty
}

\author{
A. Terrier ${ }^{\text {a,* }}$, X. Larrea ${ }^{\text {a }}$, V. Malfroy Camine ${ }^{\text {a }}$, D.P. Pioletti ${ }^{\text {a }}$, A. Farron ${ }^{\text {b }}$ \\ a Laboratory of Biomechanical Orthopedics, Ecole Polytechnique Fédérale de Lausanne, Station 19, 1015 Lausanne, Switzerland \\ b Service of Orthopaedics and Traumatology, University Hospital Center and University of Lausanne, Rue du Bugnon 46, 1011 Lausanne, Switzerland
}

\section{A R T I C L E I N F O}

\section{Article history:}

Received 24 April 2012

Accepted 15 November 2012

\section{Keywords:}

Shoulder

Rotator cuff muscles

Arthroplasty

Musculoskeletal model

\begin{abstract}
A B S T R A C T
Background: The rotator cuff muscles are the main stabilizer of the glenohumeral joint. After total shoulder arthroplasty using anterior approaches, a dysfunction of the subscapularis muscle has been reported. In the present paper we tested the hypothesis that a deficient subscapularis following total shoulder arthroplasty can induce joint instability.

Methods: To test this hypothesis we have developed an EMG-driven musculoskeletal model of the glenohumeral joint. The model was based on an algorithm that minimizes the difference between measured and predicted muscular activities, while satisfying the mechanical equilibrium of the glenohumeral joint. A movement of abduction in the scapular plane was simulated. We compared a normal and deficient subscapularis. Muscle forces, joint force, contact pattern and humeral head translation were evaluated.

Findings: To satisfy the mechanical equilibrium, a deficient subscapularis induced a decrease of the force of the infraspinatus muscle. This force decrease was balanced by an increase of the supraspinatus and middle deltoid. As a consequence, the deficient subscapularis induced an upward migration of the humeral head, an eccentric contact pattern and higher stress within the cement.

Interpretation: These results confirm the importance of the suscapularis for the long-term stability of total shoulder arthroplasty.
\end{abstract}

@ 2012 Elsevier Ltd. All rights reserved.

\section{Introduction}

After total shoulder arthroplasty (TSA), a dysfunction of the subscapularis (SC) muscle has been reported to be higher than $60 \%$ (Miller et al., 2003). It is assumed that the SC muscle deficiency can reduce the stability of the joint and the life span of the prosthesis. The SC muscle is indeed part of the rotator cuff muscles, which are the main stabilizer of the glenohumeral joint.

Computational musculoskeletal models of human joints are increasingly used to analyze normal, pathologic or prosthetic joints. They predict muscle and joint forces for a given movement, which can be used to estimate stress within bone, soft tissues and prostheses. Musculoskeletal models have to solve the indeterminacy of the mechanical system. This problem is particularly present for the shoulder, which has a wide range of motion, and many muscles. As for other joints, three techniques are used: 1) group muscles to suppress the indeterminacy (Inman et al., 1944), 2) use inverse dynamics coupled with optimization of a cost function (Charlton and Johnson, 2006; Dickerson et al., 2007; Favre et al., 2005; Holzbaur et al., 2005; Karlsson and Peterson, 1992; Terrier et al., 2010; van der Helm, 1994), or 3) use forward dynamics with electromyography (EMG) measurements as input (Langenderfer et al., 2005; Laursen et al., 1998; Terrier et al., 2008).

\footnotetext{
* Corresponding author.

E-mail address: alexandre.terrier@epfl.ch (A. Terrier).
}

Numerical shoulder models have already been used to analyze and improve TSA (Couteau et al., 2001; Gupta et al., 2004; Hopkins et al., 2007; Lacroix et al., 2000; Terrier et al., 2009). In particular, the problem of rotator cuff tears or muscle deficiencies has also been investigated with numerical tools. Because of their stabilizing function, deficiencies of the rotator cuff muscles are associated with glenohumeral instability and loosening of the glenoid component (Franklin et al., 1988; Moeckel et al., 1993; Wirth and Rockwood, 1996). The effect of rotator cuff tears on glenohumeral stability has been evaluated using an inverse dynamics model (Steenbrink et al., 2009). The authors reported that massive cuff tears beyond the supraspinatus induced an eccentric reaction force and thus joint instability. The humeral head migration was however not evaluated in this study. In another paper, an EMG-driven model predicted an increased superior migration of the humeral head when the supraspinatus muscle was deficient (Terrier et al., 2007). This EMG-driven model was however using constant muscular force ratios, which limit its application to symmetrical muscular deficiencies.

Therefore, the aim of this work was to test the hypothesis that a deficiency of the SC muscle after TSA can reduce the glenohumeral stability. To test this hypothesis, we have developed an EMG-driven musculoskeletal model of the glenohumeral joint, which allows humeral head translation. We have predicted muscle forces, joint force, humeral head translation and joint contact pattern. The latter two quantities were related to glenohumeral joint stability. 


\section{Methods}

We developed an optimization module for an existing musculoskeletal model of the glenohumeral joint allowing gleno-humeral translations (Terrier et al., 2008). This optimization module minimizes the difference between experimentally measured muscular activities and predicted ones. This updated model was used to analyze the effect of a deficiency of the SC muscle after TSA, during a movement of arm elevation in the plane of the scapula.

We used an existing musculoskeletal model to get moment arms and to predict the glenohumeral stability (Terrier et al., 2008). This model was built from a cadaveric shoulder without any sign of pathology. Bone geometry was obtained after segmentation (www. visageimaging.com) and surface reconstruction (www.geomagic. com) of computed tomography (CT) images. Muscle origins and insertions were obtained at dissection. This model includes the scapula, humerus and six scapulo-humeral muscles: middle deltoid (MD), anterior deltoid (AD), posterior deltoid (PD), supraspinatus (SS), subscapularis (SC) and infraspinatus combined with teres minoris (IS). Each muscle was modeled as a passive deformable part that could wrap around the humerus, and an active part that contained the contraction force. The IS and SC muscles were divided into three contributions of equal cross-sections and scapular origins evenly distributed on the attachment zone. The stabilization of humeral head into the glenoid fossa was achieved by the wrapping of the muscles around the humeral head and by the glenohumeral contact surfaces. This method allowed the natural translation of the humeral head into the glenoid surface. The movement of abduction on the scapular plane was controlled by an imposed shortening of the MD associated with a feedback algorithm, which continuously sets muscle forces according to predefined muscular force ratios. The scapulo-humeral rhythm was estimated from experimental measurements (McClure et al., 2001), fitted with a continuous polynomial function. The arm weight was $37.5 \mathrm{~N}$, corresponding to $5 \%$ of the bodyweight $(750 \mathrm{~N})$. The movement was realized without and with an additional weight of $10 \mathrm{~N}$ in the hand. The model was implemented in Abaqus version 6.9 (www.simulia.com). The feedback algorithm was implemented as a Fortran user subroutine within Abaqus.

The optimization module solved the quasi-static equations of mechanical equilibrium of the joint.

$\sum_{i} r_{i} \times F_{i}+\sum_{e} r_{e} \times F_{e}=0$

where $\mathbf{r}_{i}$ and $\mathbf{F}_{i}$ are respectively the moment arms and forces of the muscles, and $\mathbf{F}_{e}$ and $\mathbf{r}_{e}$ are external forces and associated moment arms, such as arm weight or additional hand load. The amplitude of the muscle forces, $F_{i}$, was related to muscle activation through the following Hill model (Zajac, 1989)

$F_{i}=k a_{i} f\left(l_{i}\right) A_{i}$

where $k$ is the Fick constant, $a_{i}$ is the activation, $f\left(l_{i}\right)$ is the isometric force-length relationship, and $A_{i}$ is the physiological cross section area (PCSA) of each muscle $i$. Without loss of generality, the muscle forces were normalized to the MD force

$\tilde{F}_{i}=\frac{F_{i}}{F_{M D}}=\tilde{a}_{i} \frac{f l_{i} A_{i}}{f_{M D} A_{M D}}$

where $\tilde{a}_{i}=a_{i} / a_{M D}$ is the normalized muscle activity.

Assuming known direction and moment arm of muscular forces, Eq. (1) is indeterminate when more than 3 muscles are included. This indeterminacy, was solved by minimizing the function

$G=\sum_{i}\left(\tilde{a}_{i}-\bar{a}_{i}\right)^{2}$ where $\bar{a}_{i}$ is a normalized experimental activation. The minimization was constrained by 2 conditions

$P\left(\tilde{a}_{i}, l_{i}, r_{i}, d_{i}\right)=0$

$\tilde{a}_{i}>0$.

The first corresponds to the projection $P$ of the angular momentum equations (Eq. (1)) into the vertical plane of motion. The external forces (vertical weight) were thus excluded from $P$. The second guarantees a positive relative activation $\tilde{a}_{i}$.

The experimental normalized activity $\bar{a}_{i}$ was derived from EMG measurements (Kronberg et al., 1990). These experimental EMG measurements were performed on 10 healthy volunteers at $0,30,60,90$, 120 and $150^{\circ}$ of abduction in the frontal plane. The original EMG measurements were normalized to the measured EMG of the middle deltoid. A piecework continuous polynomial function (cubic spline) was then fitted on the normalized EMG measurements, to get a continuous experimental normalized activation $\bar{a}_{i}$. The muscle parameters introduced in Eq. (2) were adapted from the literature (Table 1).

The musculoskeletal model provided the moment arms $\mathbf{r}_{i}$, the force direction $\mathbf{d}_{i}$, and the muscle length $l_{i}$. During the quasi-static abduction of the arm, the optimization algorithm was thus fully integrated as a Fortran subroutine into the iterative solver of Abaqus. The integration of the optimization algorithm into Abaqus guaranteed the mechanical equilibrium of the joint and the optimization of the criteria at each converged iteration of the iterative solving process. The FE solver used variable angle increments to perform arm motion. For each increment, several iterations might be required to solve the non-linear problem. For each iteration, the solver builds the stiffness matrix. This matrix is partly composed of the muscle elements. During this process, the FE solver calls the optimization algorithm, which is a Fortran user-defined element (UEL) subroutine. This subroutine also calls different other subroutines and controls the muscle synchronization (Fig. 1). The muscle moment arms and length are calculated, to perform the constrained minimization of Eqs. (5) and (6). This gives the muscular ratios, which can be used to predict the muscular force. The force and length variation of the muscles can be obtained to build their contribution to the stiffness matrix. An algebraic form was already obtained for this contribution to the stiffness matrix (Terrier et al., 2008). The polynomial fit to experimental data was achieved by CUBSPL and PPVALU functions of the PPPACK library (www.netlib.org/pppack). The solving of the constrained optimization was achieved by DBOCLS function of the DQED library (www. netlib.org/opt).

The Aequalis prosthesis (Tornier, Inc., Edina, MN, USA) was positioned in the numerical model by a senior orthopedic surgeon according to the manufacturer recommendations. The humeral head component radius was $24 \mathrm{~mm}$ and the glenoid component radius of curvature was $30 \mathrm{~mm}$. The polyethylene glenoid component was assumed elastic $(\mathrm{E}=500 \mathrm{MPa}, \nu=0.4)$, and the metallic humeral head and bone were considered rigid.

The model was first tested with a normal muscular function, without and with $10 \mathrm{~N}$ in the hand. Then, two cases were compared: TSA with a normal muscular function and TSA with a deficiency of the SC. For this comparison, $10 \mathrm{~N}$ was added in the hand. We simulated the

Table 1

Muscle properties of the model. $L_{O P T}, L_{S L}$ and $A$ are the optimal muscle length, the tendon slack length, and the physiological cross section area (Gollapudi and Lin, 2009; Hill, 1938; Langenderfer et al., 2004; Zajac, 1989).

\begin{tabular}{lcccccc}
\hline & MD & AD & PD & SS & SC & IS \\
\hline$L_{\text {OPT }}[\mathrm{mm}]$ & 107.2 & 107.1 & 150.2 & 67.3 & 104.3 & 88.1 \\
$L_{S L}[\mathrm{~mm}]$ & 12.8 & 21.5 & 33.1 & 24.4 & 29.5 & 42.0 \\
$A\left[\mathrm{~cm}^{2}\right]$ & 7.39 & 5.46 & 4.69 & 3.36 & 9.49 & 8.34 \\
\hline
\end{tabular}




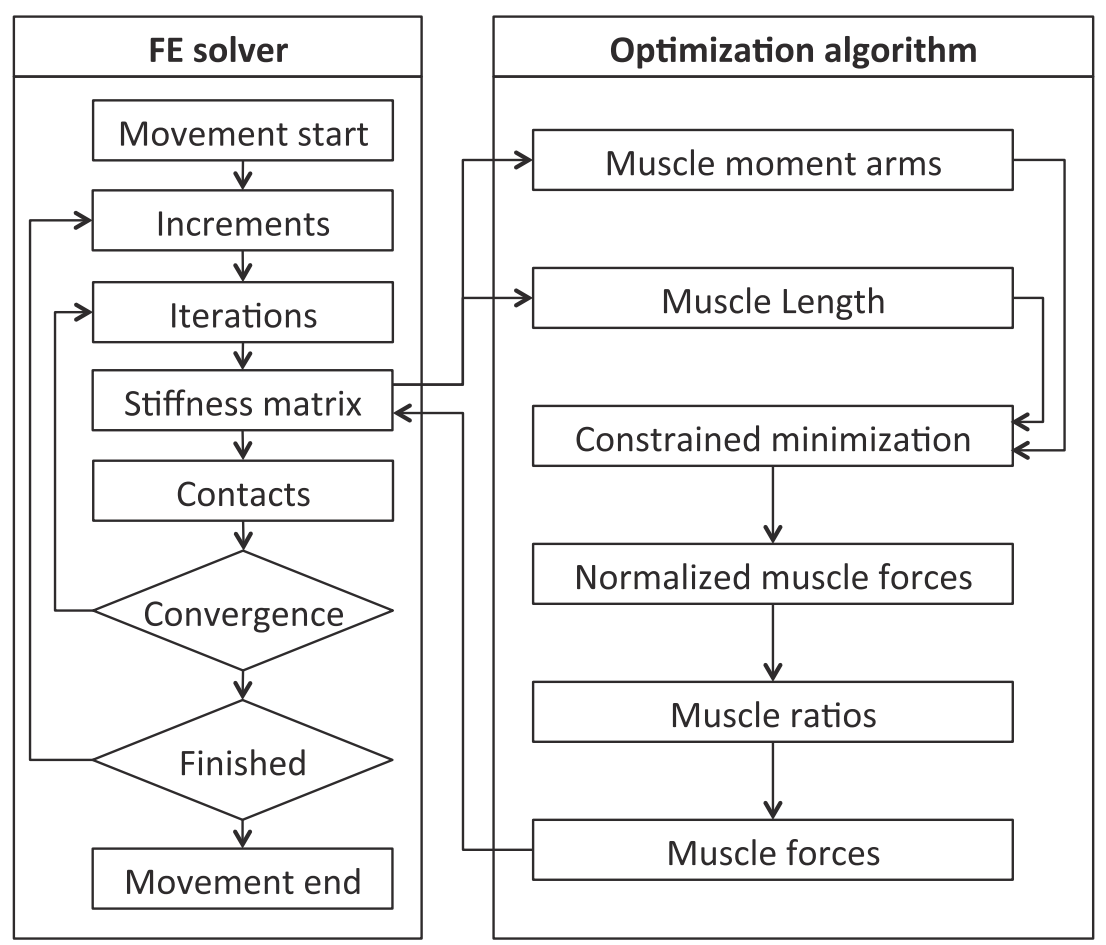

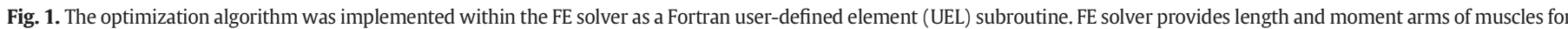
the constrained minimization, which finally returns muscular forces to build the FE stiffness matrix. The subroutine is called at each iteration of the non-linear FE solver.

deficiency of the SC by reducing its PCSA to $50 \%$ of the normal value. We evaluated the muscle forces, the joint force, the humeral translation, and the glenohumeral contact pattern.

\section{Results}

With a normal muscular function and no additional load in the hand, the maximal forces of the MD, $\mathrm{AD}, \mathrm{PD}, \mathrm{SS}, \mathrm{SC}$, and IS were respectively $226,99,74,72,88$ and $94 \mathrm{~N}$. The joint force reached $567 \mathrm{~N}$ at $83^{\circ}$ of abduction (Fig. 2). With an additional weight of $10 \mathrm{~N}$ in the hand, the muscle and glenohumeral forces increased by $57 \%$.

In the case of 50\% SC deficiency, the maximal force of SC was reduced by $43 \%$ (Fig. 3). It also induced a decrease of $26 \%$ of the IS force, but an overall increase of the MD, AD, PD and SS of $6 \%$. The

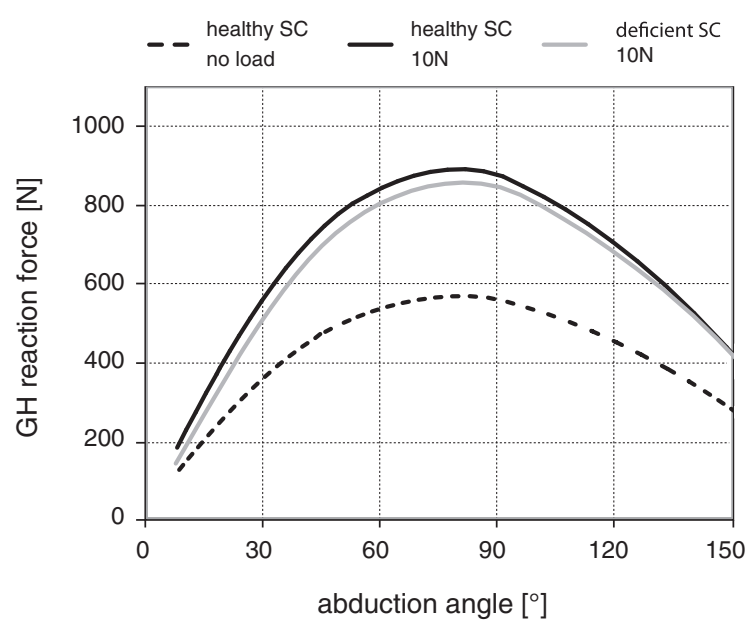

Fig. 2. Glenohumeral $(\mathrm{GH})$ reaction force during abduction, with healthy $\mathrm{SC}$ and no load in the hand (healthy SC/0 N), with healthy SC and $10 \mathrm{~N}$ in the hand (healthy SC/10 N), and with deficient SC and $10 \mathrm{~N}$ in the hand (deficient SC/10 N). maximal glenohumeral force on the glenoid was $4 \%$ lower and was oriented in a more postero-superior direction.

With a normal muscular function, the humeral head translation was about $3 \mathrm{~mm}$ and mainly directed upward (Fig. 4). The contact pattern followed an up and down movement (Fig. 3). The average and local pressure on the glenoid component reached 8 and $18 \mathrm{MPa}$ respectively. An additional weight of $10 \mathrm{~N}$ in the hand had little effect on the humerus translation $(0.15 \mathrm{~mm})$, but the average and local contact pressure increased by $20 \%$ and $26 \%$ respectively.

A deficiency of the SC muscle increased the displacement of the humeral head by $0.5 \mathrm{~mm}$ in the superior direction, and by $0.1 \mathrm{~mm}$ in the posterior direction. The contact pattern was more eccentric (postero-superior) and smaller $\left(54 \mathrm{~mm}^{2}\right.$ vs. $57 \mathrm{~mm}^{2}$ ). The average and local contact pressure decreased by $2 \%$.

\section{Discussion}

Anterior approaches for TSA can produce a dysfunction of the subscapularis muscle. We tested here the hypothesis that this deficiency can induce a glenohumeral joint instability. To test this hypothesis, we developed an EMG-driven model that minimizes the difference between measured and predicted muscular activities. The joint stability was achieved by the muscles, which allowed the natural translation of the humeral head. This feature was critical to analyze the effect of rotator cuff deficiency on joint stability after TSA. The numerical model predicted that a deficient subscapularis resulted not only in a lower subscapularis force, but also in a lower infraspinatus force and higher forces in the middle deltoid and anterior deltoid. This pathological muscular function reduced the joint stability, and increased the humeral head migration and the contact pressure on the glenoid component.

This EMG-driven model predicted muscular forces that best match relative muscular activities measured experimentally on 6 scapulohumeral muscles. With a healthy SC, the additional weight of $10 \mathrm{~N}$ in the hand induced higher muscle and joint forces, but did not change the translation of the humeral head. To simulate an atrophied SC muscle, its PCSA was reduced by $50 \%$. The SC force was however only 


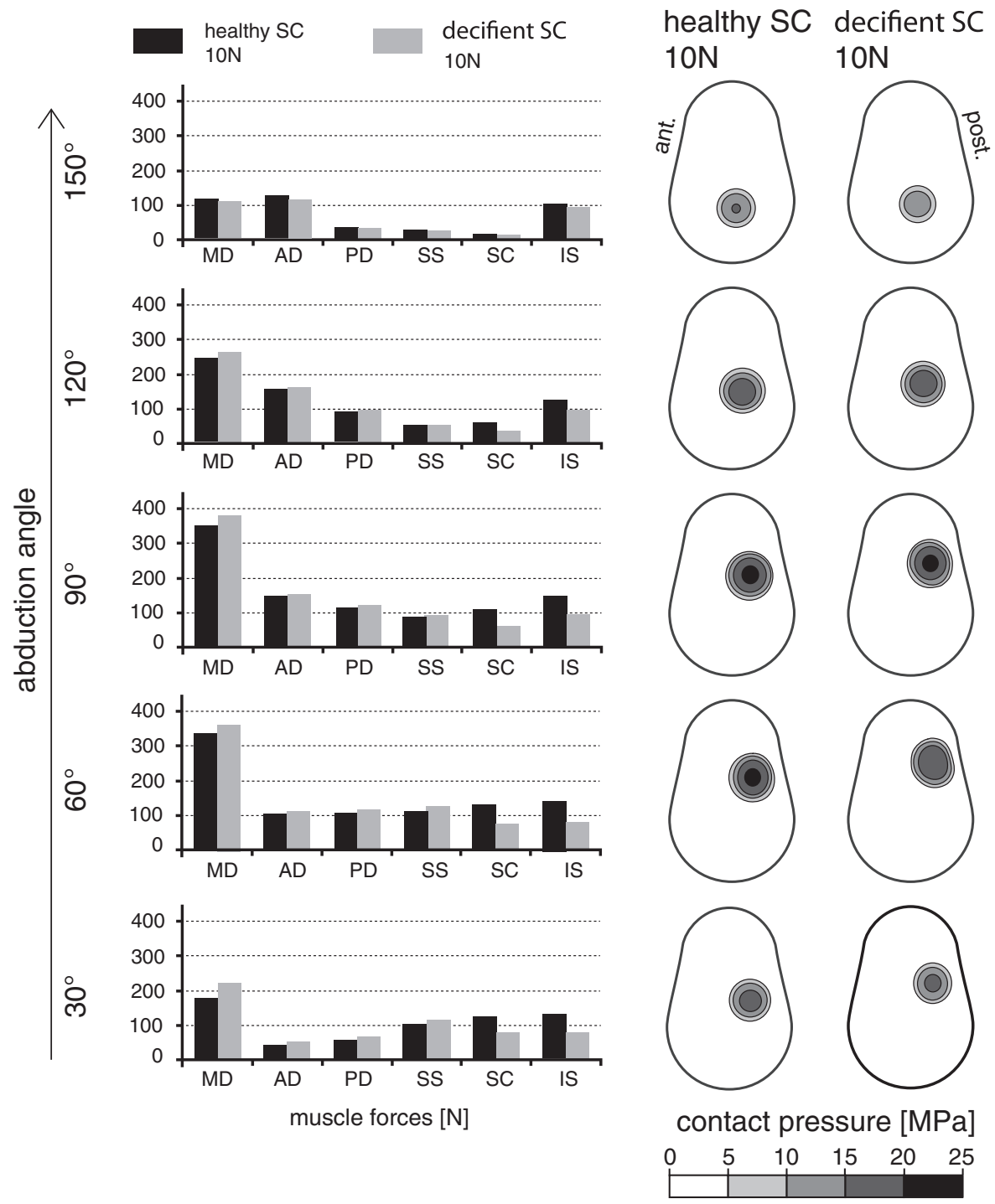

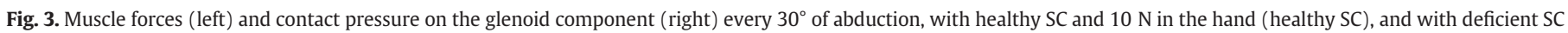
and $10 \mathrm{~N}$ in the hand (deficient SC).

reduced by $43 \%$. The optimization algorithm actually predicted a higher SC muscle activity to compensate for the PCSA decrease. The decrease of the $\mathrm{SC}$ force induced a balanced decrease of the IS force to avoid a torque that would induce out of plane elevation of the arm. The loss of elevation torque related to SC and IS force decrease was balanced by a force increase of the other muscles, mainly the MD, AD, PD, and SS. Globally, this produced to a more vertical resultant force on the humerus. The
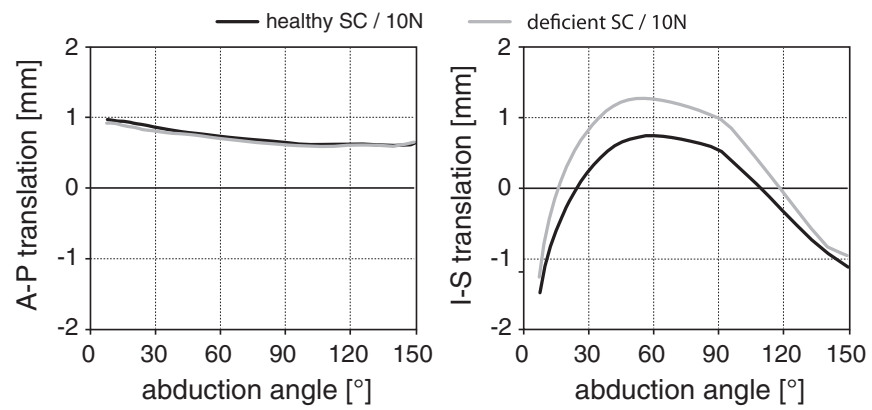

Fig. 4. Antero-posterior (left) and infero-superior (right) translation of the humeral head during abduction, with healthy SC and $10 \mathrm{~N}$ in the hand (healthy SC), and with deficient SC and $10 \mathrm{~N}$ in the hand (deficient SC). more eccentric contact trajectory towards the posterior-superior edge of the implant resulted in a slightly smaller contact area. Since the deficiency of the SC also slightly decreased the reaction force, the effect on the contact pressure was negligible. The muscle moment arms were not presented here, as they were the same as with a preliminary model (Terrier et al., 2007).

Compared to other musculoskeletal models, the present method predicted higher joint reaction forces than optimization based models but similar to EMG-driven models. As an indirect validation, the present results compared well with in vivo measurements (Bergmann et al., 2007). At 45 and $75^{\circ}$ of abduction, the measured glenohumeral force was $51 \%$ and $85 \%$ of the body weight, which indeed correspond well with our predictions ( $63 \%$ and $76 \%$ of $750 \mathrm{~N}$ assumed in this study). Previous numerical work relating SC deficiency and glenohumeral stability also predicted a more supero-posterior direction of the reaction force (Steenbrink et al., 2009), which is consistent with the eccentric contact pattern shown by our model. With a normal muscle function, the natural humeral head translation has been reported to be in the range from $1 \mathrm{~mm}$ to $5 \mathrm{~mm}$ (Deutsch et al., 1996; Graichen et al., 2000; Kelkar et al., 2001; Wuelker et al., 1994; Yamaguchi et al., 2000). A higher humeral head translation has already been related to deficient rotator cuff musculature. In vitro biomechanical models (Kelkar et al., 2001; Konrad et al., 2006; Mura et al., 2003; Wuelker et al., 1994) and in vivo studies 
(Deutsch et al., 1996; Graichen et al., 2000; Yamaguchi et al., 2000)

showed similar results as the ones predicted here.

The main strength of this model is its ability to replicate the muscular stabilization of the joint, allowing the natural translation of the humeral head. An iterative process simultaneously calculated the muscle forces required for the motor and stabilizing action. Relative activity and forces were used to extrapolate EMG measurements to different loading amplitudes, assuming that the activity pattern remains the same when the movement is the same. This is actually the main limitation of the present model, which indeed requires EMG data for a specific movement. The EMG was measured during an abduction movement in the frontal plane, while we are simulating a movement in the scapular plane. However, according to another EMG experimental study, we assumed that the muscle activation patterns are rather similar for these two planes of elevation (Ringelberg, 1985). Moreover, the muscle force ratios for the deficient SC case were calculated with the same healthy EMG data. Ideally EMG data from deficient SC subjects would be desirable, however, as a first approximation, and since only a $50 \%$ SC deficiency was simulated, we assumed that healthy EMG data could be used. This approximation was supported by an EMG study comparing healthy subjects to rotator cuff tear patients and reporting no significant differences in inter-muscular coordination (Hawkes et al., 2012). We associated muscular deficiency with PCSA, as suggested by clinical observations (De Wilde et al., 2012; Goutallier et al., 1994). The PCSA decrease was set to $50 \%$ as a first estimate, but in a next step this value will be measured directly on the patient CT.

\section{Conclusion}

The model confirms the hypothesis that a deficient SC muscle can reduce the long-term stability of the glenoid component after TSA. The loss of stability induces the "rocking horse" effect that can lead to a progressive loosening of the glenoid component. This study confirms that a particular attention must be paid to repair strongly the subscapularis tendon after TSA. Moreover this type of implants should probably not be used in subscapularis deficient shoulders.

\section{Conflict of interest}

None of the authors has any conflict of interest.

\section{Acknowledgments}

This project was supported by the Inter-Institutional Center for Translational Biomechanics (EPFL-CHUV-DAL) and Tornier (Tornier, Inc., Edina, MN).

\section{References}

Bergmann, G., Graichen, F., Bender, A., Kaab, M., Rohlmann, A., Westerhoff, P., 2007. In vivo glenohumeral contact forces-Measurements in the first patient 7 months postoperatively. J. Biomech. 40 (10), 2139-2149.

Charlton, I.W., Johnson, G.R., 2006. A model for the prediction of the forces at the glenohumeral joint. Proc. Inst. Mech. Eng. H 220 (8), 801-812.

Couteau, B., Mansat, P., Estivalezes, E., Darmana, R., Mansat, M., Egan, J., 2001. Finite element analysis of the mechanical behavior of a scapula implanted with a glenoid prosthesis. Clin. Biomech. 16 (7), 566-575.

De Wilde, L.F., De Coninck, T., De Neve, F., Berghs, B.M., 2012. Subscapularis release in shoulder replacement determines structural muscular changes. Clin. Orthop. Relat. Res. 470 (8), 2193-2201.

Deutsch, A., Altchek, D.W., Schwartz, E., Otis, J.C., Warren, R.F., 1996. Radiologic measurement of superior displacement of the humeral head in the impingement syndrome. J. Shoulder Elbow Surg. 5 (3), 186-193.

Dickerson, C.R., Chaffin, D.B., Hughes, R.E., 2007. A mathematical musculoskeletal shoulder model for proactive ergonomic analysis. Comput. Methods Biomech. Biomed. Engin. 10 (6), 389-400.

Favre, P., Sheikh, R., Fucentese, S.F., Jacob, H.A., 2005. An algorithm for estimation of shoulder muscle forces for clinical use. Clin. Biomech. 20 (8), 822-833.

Franklin, J.L., Barrett, W.P., Jackins, S.E., Matsen III, F.A., 1988. Glenoid loosening in total shoulder arthroplasty. Association with rotator cuff deficiency. J. Arthroplasty 3 (1), 39-46.
Gollapudi, S.K., Lin, D.C., 2009. Experimental determination of sarcomere force-length relationship in type-I human skeletal muscle fibers. J. Biomech. 42 (13), 2011-2016.

Goutallier, D., Postel, J.M., Bernageau, J., Lavau, L., Voisin, M.C., 1994. Fatty muscle degeneration in cuff ruptures. Pre- and postoperative evaluation by CT scan. Clin. Orthop. Relat. Res. (304), 78-83.

Graichen, H., Stammberger, T., Bonel, H., Karl-Hans, E., Reiser, M., Eckstein, F., 2000. Glenohumeral translation during active and passive elevation of the shoulder - a 3D open-MRI study. J. Biomech. 33 (5), 609-613.

Gupta, S., van der Helm, F.C., van Keulen, F., 2004. Stress analysis of cemented glenoid prostheses in total shoulder arthroplasty. J. Biomech. 37 (11), 1777-1786.

Hawkes, D.H., Alizadehkhaiyat, O., Kemp, G.J., Fisher, A.C., Roebuck, M.M., Frostick, S.P. 2012. Shoulder muscle activation and coordination in patients with a massive rotator cuff tear: an electromyographic study. J. Orthop. Res. 30 (7), 1140-1146.

Hill, A.V., 1938. The heat of shortening and the dynamic constants of muscle. Proc. R. Soc. Lond. B Biol. Sci. 126 (843), 136-195.

Holzbaur, K.R., Murray, W.M., Delp, S.L., 2005. A model of the upper extremity for simulating musculoskeletal surgery and analyzing neuromuscular control. Ann. Biomed. Eng. 33 (6), 829-840.

Hopkins, A.R., Hansen, U.N., Amis, A.A., Taylor, M., Emery, R.J., 2007. Glenohumeral kinematics following total shoulder arthroplasty: a finite element investigation. J. Orthop. Res. 25 (1), 108-115.

Inman, V.T., Saunders, J.B., Abbott, L.C., 1944. Observations of the function of the shoulder joint. J. Bone Joint Surg. Am. (26), 1-30.

Karlsson, D., Peterson, B., 1992. Towards a model for force predictions in the human shoulder. J. Biomech. 25 (2), 189-199.

Kelkar, R., Wang, V.M., et al., 2001. Glenohumeral mechanics: a study of articular geometry contact, and kinematics. J. Shoulder Elbow Surg. 10 (1), 73-84.

Konrad, G.G., Markmiller, M., et al., 2006. Decreasing glenoid inclination improves function in shoulders with simulated massive rotator cuff tears. Clin. Biomech. 21 (9), 942-949.

Kronberg, M., Nemeth, G., Brostrom, L.A., 1990. Muscle activity and coordination in the normal shoulder. An electromyographic study. Clin. Orthop. Relat. Res. (257), 76-85.

Lacroix, D., Murphy, L.A., Prendergast, P.J., 2000. Three-dimensional finite element analysis of glenoid replacement prostheses: a comparison of keeled and pegged anchorage systems. J. Biomech. Eng. 122 (4), 430-436.

Langenderfer, J., Jerabek, S.A., Thangamani, V.I., Kuhn, J.E., Hughes, R.E., 2004. Musculoskeletal parameters of muscles crossing the shoulder and elbow and the effect of sarcomere length sample size on estimation of optimal muscle length. Clin. Biomech. 19 (7), 664-670.

Langenderfer, J., LaScalza, S., Mell, A., Carpenter, J.E., Kuhn, J.E., Hughes, R.E., 2005. An EMG-driven model of the upper extremity and estimation of long head biceps force. Comput. Biol. Med. 35 (1), 25-39.

Laursen, B., Jensen, B.R., Nemeth, G., Sjogaard, G., 1998. A model predicting individual shoulder muscle forces based on relationship between electromyographic and 3D external forces in static position. J. Biomech. 31 (8), 731-739.

McClure, P.W., Michener, L.A., Sennett, B.J., Karduna, A.R., 2001. Direct 3-dimensional measurement of scapular kinematics during dynamic movements in vivo. J. Shoulder Elbow Surg. 10 (3), 269-277.

Miller, S.L., Hazrati, Y., Klepps, S., Chiang, A., Flatow, E.L., 2003. Loss of subscapularis function after total shoulder replacement: A seldom recognized problem. J. Shoulder Elbow Surg. 12 (1), 29-34.

Moeckel, B.H., Altchek, D.W., Warren, R.F., Wickiewicz, T.L., Dines, D.M., 1993. Instability of the shoulder after arthroplasty. J. Bone Joint Surg. Am. 75 (4), 492-497.

Mura, N., O'Driscoll, S.W., et al., 2003. The effect of infraspinatus disruption on glenohumeral torque and superior migration of the humeral head: a biomechanical study. J. Shoulder Elbow Surg. 12 (2), 179-184.

Ringelberg, J.A., 1985. EMG and force production of some human shoulder muscles during isometric abduction. J. Biomech. 18 (12), 939-947.

Steenbrink, F., de Groot, J.H., Veeger, H.E., van der Helm, F.C., Rozing, P.M., 2009. Glenohumeral stability in simulated rotator cuff tears. J. Biomech. 42 (11), 1740-1745

Terrier, A., Reist, A., Vogel, A., Farron, A., 2007. Effect of supraspinatus deficiency on humerus translation and glenohumeral contact force during abduction. Clin. Biomech. 22 (6), 645-651.

Terrier, A., Vogel, A., Capezzali, M., Farron, A., 2008. An algorithm to allow humerus translation in the indeterminate problem of shoulder abduction. Med. Eng. Phys. 30 (6), 710-716.

Terrier, A., Merlini, F., Pioletti, D.P., Farron, A., 2009. Total shoulder arthroplasty: downward inclination of the glenoid component to balance supraspinatus deficiency. J. Shoulder Elbow Surg. 18 (3), 360-365.

Terrier, A., Aeberhard, M., et al., 2010. A musculoskeletal shoulder model based on pseudo-inverse and null-space optimization. Med. Eng. Phys. 32 (9), 1050-1056.

van der Helm, F.C., 1994. A finite element musculoskeletal model of the shoulder mechanism. J. Biomech. 27 (5), 551-569.

Wirth, M.A., Rockwood Jr., C.A., 1996. Complications of total shoulder-replacement arthroplasty. J. Bone Joint Surg. Am. 78 (4), 603-616.

Wuelker, N., Schmotzer, H., Thren, K., Korell, M., 1994. Translation of the glenohumeral joint with simulated active elevation. Clin. Orthop. Relat. Res. (309), 193-200.

Yamaguchi, K., Sher, J.S., et al., 2000. Glenohumeral motion in patients with rotator cuff tears: a comparison of asymptomatic and symptomatic shoulders. J. Shoulder Elbow Surg. 9 (1), 6-11.

Zajac, F.E., 1989. Muscle and tendon: properties, models, scaling, and application to biomechanics and motor control. Crit. Rev. Biomed. Eng. 17 (4), 359-411. 